\title{
LIFE AS A DENTAL CORE TRAINEE: ORAL SURGERY
}

\section{By Adam Shathur}

started my Dental Core Training (DCT) 1 post in September 2019 at St George's University Hospital, London. The post is separated across two different hospitals, St. George's Hospital and Kingston Hospital. At present, I am nearing the end of my rotation in St. George's and look forward to starting in Kingston Hospital. The position I have undertaken is a year of further education predominantly dedicated to Oral Surgery, the only one of its kind in England. So what's it like?

During the time situated in St George's, the post consists of two main components, consultation clinics and operative sessions. The clinics include complex Oral Surgery/Medicine referrals and Oral and Maxillofacial Surgery (OMFS) consisting of two major branches: deformity and oncology. Every clinical session is led by different consultants alongside their registrar, ensuring exposure and the opportunity to work alongside numerous prestigious clinicians each week.

\section{Consultation Clinics}

St George's is a well-renowned large London hospital, due to this we are often referred rare conditions which you may not otherwise come across throughout your career as a GDP. Working alongside world class surgeons with the challenge of interesting and unique cases each day inspires you to further your own knowledge of these presentations and how they should be managed.

Working at St. George's OMFS department offers the particular advantage of Restorative, Paediatric, Orthodontic, Radiology departments and even the OMFS technicians located on the same floor. This works efficiently as often patients require a multidisciplinary approach due to the complex nature of their treatment. It also offers the advantage should you require a specialist's opinion from another department, then it is easy to access. This integration of departments also benefits the training for DCTs as often we have the opportunity to learn from different specialties. An example of this is when
I was given the opportunity to work alongside a restorative consultant for a large hypodontia case and was able to place a dental implant myself only three months into my post.

There are also hands-on procedures carried out during the clinic sessions, such as the removal of Leonard Buttons used for intermaxillary fixation and the debridement of wounds and lacerations. Additionally, a vast number of clinics involve the administration of botulinum toxin to help manage presentations such as sialorrhea and temporomandibular joint dysfunction (TMD).

\section{'Working as part of a multi- disciplinary team in a hospital environment has a range of advantages'}

\section{Operative Sessions}

There are a range of practical aspects key to this DCT position. A vast majority of patients present with complex tier 3 exodontia cases such as horizontally impacted mandibular third molars which are considered too complex for removal by a GDP. There are also a range of exodontia cases which are referred to our care due to complex medical histories thus we are required to liaise with our medical colleagues to ensure the safe treatment of these patients in a secondary setting. Furthermore, we often carry out incisional and excisional biopsies for numerous pathologies ranging from fibroepithelial polyps and mucoceles, to lesions of suspected malignancy. Along side these, we often have the opportunity to complete cyst enucleations and superficial salivary stone removals.

As a DCT I am also often assisting in theatre where more complex procedures are carried out.
Prior to theatre, is it my responsibility to prepare the list of patients who will be undergoing surgery by liaising with the anaesthetist in preparation for the management of any medical problems, ensuring all of the relevant images such as radiographs/scans are available and by ensuring a clear correct treatment plan is prepared. Once in theatre, we scrub up as for any other surgery and work under strict infection control standards. As well as extractions, exposing unerupted teeth, removing infected plates and soft tissue biopsies, I have had the pleasure of assisting OMFS consultants for procedures including mandibular bone biopsies for suspected malignancy, and have had the opportunity to carry out arthrocentesis for management of TMD.

After my 6 month rotation at St George's I will begin my subsequent 6 months in Kingston. Kingston is a district general hospital, meaning that usually the departments work in smaller teams. Therefore, I will have more independence and further responsibility with more operative sessions. In addition, I will be on-call during the day for the accident and emergency unit in the hospital for all OMFS admissions. Furthermore, I will be working in the Restorative department regularly dealing with complex restorative treatment plans and medical histories which require a hospital environment.

As a DCT I am given weekly tutorial sessions varying from a range of topics which allows me to further my knowledge and provides guidance in certain subjects. I am also encouraged to carry out audits with the potential prospect of publications, and to undergo further qualifications and courses to widen my skills and improve my professional portfolio.

DCT has been invaluable and rewarding thus far and I encourage all prospective students to consider this year of further training. Working as part of a multi-disciplinary team in a hospital environment has a range of advantages, the complexity and uniqueness of the cases seen enable you to use all of the skills acquired during undergraduate training and reach your potential as a clinician. Every day is a new challenge, and you will be faced with new opportunities.

Adam Shathur

https://doi.org/10.1038/s41406-020-0159-1 